\title{
Combined Intra- and Inter- Block Analysis of Balanced Ternary Designs
}

\author{
Sobita Sapam \\ Guest Lecturer, Department of Statistics, Manipur University, \\ Canchipur, Imphal-795003, India \\ sobital@yahoo.com \\ B. K. Sinha \\ ISI, Kolkata [Retired Professor of Statistics] \\ 203, B. T. Road, Kolkata- 700 108, India \\ bikassinha1946@gmail.com \\ N.K. Mandal \\ Professor of Statistics (Retired), Calcutta University \\ 35, B.C. Road, Kolkata-700 019, India \\ mandalnk2001@yahoo.co.in
}

Received 31 December 2016

Accepted 29 March 2017

\begin{abstract}
In the present paper an attempt has been made to study the inter-and intrablock estimation of treatment effects contrasts with random block effects in the context of balanced ternary designs. The analysis is illustrated through examples.
\end{abstract}

Keywords: Balanced ternary design; Intra-block analysis; Inter-block analysis; Combined intra- and inter-block analysis.

2000 Mathematics Subject Classification: 62K10 


\section{Introduction}

Balanced n-ary designs were introduced by Tocher (1952). In such a design each entry in the incidence matrix can take any value of the ' $n$ ' possible values, usually, $0,1,2, . .,(n-1)$. If $n=3$, we get a ternary design. Billington(1984,1989), Meena and Mangla(2006), Sharma, Singh \& Roshni(2013) give results on the construction of balanced ternary designs.

In the present paper an attempt has been made to study the inter-and intra-block estimation of treatment effects contrasts with random block effects in the context of balanced ternary designs. The term recovery of inter-block information was first coined by Yates (1939). Generally, the experimental units are partitioned to eliminate the heterogeneity of the experimental units (eus) in such a way that the eus within blocks are as homogeneous as possible. Because of the variability of the block totals, the initial estimates of the treatment contrasts were based on contrasts within blocks neglecting the information contained in block totals. If, however, the block totals are not that heterogeneous, the loss of information on treatment contrasts can be recovered by treating block totals as observations. According to Rao(1947), "If the ratio of the variances for inter-and intra-block comparisons is sufficiently greater than 1, these estimates from incomplete block designs will, of course, be considerably more accurate."

Bose(1975) worked on combined intra-and inter-block estimation of treatment effects in incomplete block designs.Searle(1986) dealt with the case of block effects being treated as random;estimators of treatment effects contrasts were derived, along with their variances, and the results were applied to balanced incomplete blocks to yield an expression for the inter-and intrablock estimator of a treatment effects contrast.

The organization of the paper is as follows: In Section 2, we introduce some useful definitions and preliminaries. Next in Section 3, we deal with two useful models viz., Fixed Effects Additive Model [FEAM] and Mixed Effects Additive Model [MEAM] in the context of Ternary Designs. The data analysis under these two models are carried out separately in this section. In section 4, combined data analysis is presented.

\section{Definitions and Preliminaries}

A Balanced Ternary Design (BTD) with parameters [v,b,r, k,t,s, $q, \rho_{1}, \rho_{q}, f_{11}, f_{1 q}$ and $f_{q q}$ ] is an arrangement of ' $\mathrm{v}$ ' treatments in ' $\mathrm{b}$ ' blocks - each of size ' $\mathrm{k}$ '- whose incidence matrix is given by $\mathbf{N}=\left(n_{i j}\right)$, where $n_{i j}$ is the number of times the $\mathrm{i}$-th treatment occurs in the $\mathrm{j}$-th block and $n_{i j}=0,1, \mathrm{q}(\geq 2)$ and the elements of the matrix $\mathbf{N}_{\mathbf{v} \times \mathbf{b}}=\left(n_{i j}\right)$ satisfy the following conditions:

(i) $n_{i j}=0$, if the $\mathrm{i}$-th treatment does not occur in the $\mathrm{j}$-th block,

$=1$, if the $\mathrm{i}$-th treatment occurs in the $\mathrm{j}$-th block exactly once,

$=q$, if the $i$-th treatment occurs in the $j$-th block exactly $q(\geq 2)$ times;

(ii) every treatment is replicated $r$ times in the entire design;

(iii) each block contains a set of ' $t$ ' treatments exactly once each and another distinct set of ' $s$ ' treatments exactly qtimes each so that $\mathrm{k}=\mathrm{t}+\mathrm{qs}, \mathrm{t}+\mathrm{s}<\mathrm{v}$;

(iv) $\rho_{1}$ is the number of blocks that any arbitrarily specified treatment occurs once in each such block; 
(v) $\rho_{q}$ is the number of blocks that any arbitrarily specified treatment occurs q times in each such block;

(vi) $f_{11}$ is the number of blocks that any pair of arbitrarily specified treatments occur together once each exactly in each of these blocks;

(vii) $f_{1 q}$ is the number of blocks that any arbitrarily specified treatment occurs once and any other arbitrarily specified treatment occurs $q(\geq 2)$ times in each of these blocks;

(viii) $f_{q q}$ is the number of blocks that any two arbitrarily specified treatments occur each q times in each of these blocks.

Then it is seen that the design parameters $\left[\mathrm{v}, \mathrm{b}, \mathrm{r}, \mathrm{k}, \mathrm{t}, \mathrm{s}, q, \rho_{1}, \rho_{2}, f_{11}, f_{1 q}\right.$ and $\left.f_{q q}\right]$ satisfy the following conditions:

(ix) bk=vr, (x) $\mathrm{r}=\rho_{1}+\mathrm{q} \rho_{q}$, (xi) $\mathrm{k}=\mathrm{t}+\mathrm{qs}$, (xii) $\rho_{1}(\mathrm{k}-1)=(\mathrm{v}-1)\left(f_{11}+f_{1 q}\right)$,

(xiii) $\rho_{q}(\mathrm{k}-\mathrm{q})=(\mathrm{v}-1)\left(f_{1 q}+q f_{q q}\right), \mathrm{q}(\geq 2)$.

Further, it follows that

(xiv) $\lambda=f_{11}+2 q f_{1 q}+q^{2} f_{q q}=\sum_{j=1}^{b} n_{i j} n_{i^{\prime} j}$ for $i \neq i^{\prime}=1,2, \ldots, \mathrm{v}$,

(xv) $\Delta=\rho_{1}+q^{2} \rho_{q}=\sum_{j=1}^{b} n_{i j}^{2}$ for each $i=1,2, \ldots, \mathrm{v}$,

(xvi) $\mathbf{N N}^{\prime}=\left[\begin{array}{ccc}\Delta & \cdots & \lambda \\ \vdots & \ddots & \vdots \\ \lambda & \cdots & \Delta\end{array}\right]$.

\section{The FEAM \& MEAM Models and ANOVA Tables}

\subsection{Mixed Effects Additive Model [MEAM] and Related ANOVA Table}

First we consider Linear MEAM. Let $y_{i j u}$ be the observation corresponding to u-th experimental unit in the $\mathrm{j}$-th block to which the $\mathrm{i}$-th treatment is applied. We then stipulate the following model

$$
y_{i j u}=\mu+\tau_{\mathrm{i}}+a_{j}+e_{i j u} ; \mathrm{i}=1,2, \ldots, \mathrm{v} ; \mathrm{j}=1,2, \ldots, \mathrm{b} ; \mathrm{u}=1,2, \ldots, \mathrm{k}
$$

where $\mu$ is the general mean effect, $\tau_{i}$ is the fixed treatment effect, $a_{j}$ is the random block effect

with mean zero and variance $\sigma_{b}^{2}, e_{i j u}$ 's are random errors assumed to be iid with mean zero and variance $\sigma_{e}^{2}$. The random errors $e_{i j u}$ 's and the random block effects $a_{j}$ 's are supposed to be uncorrelated and normally distributed.

Under MEAM, we want to explore the contribution of block average contrasts towards estimation of treatment effects contrasts. These are referred to as 'inter-block' estimates of treatment contrasts. Therefore the source of information on treatment contrasts can be obtained from the (b1) block mean contrasts. Besides these, the within block observational contrasts are known to yield 'intra-block' estimates of treatment effects contrasts. 


\section{Notations:}

Denote by $\bar{B}_{1}, \ldots, \bar{B}_{b}$ the block means for b blocks of the design. Next, we introduce elementary block mean contrasts below. Since the blocks are proper and binary, under a mixed effects model, the block mean contrasts will involve only the treatment effects contrasts in their model expectations. This will provide information [and related inference] on treatment effects in terms of their contrasts. This is referred to as Inter-Block Analysis. We start as follows:

$D_{1}=\bar{B}_{1}-\bar{B}_{2}, \ldots, D_{b-1}=\bar{B}_{1}-\bar{B}_{b}$, where $\boldsymbol{D}=\left(D_{1}, \ldots, D_{b-1}\right)^{\prime}$ which follows $N_{b-1}(\zeta, \Sigma(\boldsymbol{D}))$ with $\zeta_{j^{\prime}-1}=\mathrm{E}\left(D_{j^{\prime}-1}\right)=\mathrm{E}\left(\bar{B}_{1}-\bar{B}_{j^{\prime}}\right), j^{\prime}=2,3, \ldots$, b.

Explicitly,

$\mathrm{E}\left(D_{1}\right)=\mathrm{E}\left(\bar{B}_{1}-\bar{B}_{2}\right)=\frac{1}{k}\left[\sum_{i=1}^{v} n_{i 1} \tau_{i}-\sum_{i=1}^{v} n_{i 2} \tau_{i}\right]$

$=\frac{1}{k}\left[\sum_{i=1}^{v}\left(n_{i 1}-n_{i 2}\right) \tau_{i}\right]$

and so on;

$\mathrm{E}\left(D_{b-1}\right)=\mathrm{E}\left(\bar{B}_{1}-\bar{B}_{b}\right)=\frac{1}{k}\left[\sum_{i=1}^{v} n_{i 1} \tau_{i}-\sum_{i=1}^{v} n_{i b} \tau_{i}\right]=\frac{1}{k}\left[\sum_{i=1}^{v}\left(n_{i 1}-n_{i b}\right) \tau_{i}\right]$

Since block sizes are same, it is clear from the above expression that $\mathrm{E}\left(D_{i}\right)$ is a treatment contrast for each i.

$\operatorname{Var}\left(D_{1}\right)=\operatorname{Var}\left(\bar{B}_{1}-\bar{B}_{2}\right)=\operatorname{Var}\left(\bar{B}_{1}\right)+\operatorname{Var}\left(\bar{B}_{2}\right)$ [since all the blocks are independent]

$=\frac{2}{k^{2}}\left(k^{2} \sigma_{b}^{2}+k \sigma_{e}^{2}\right)\left[\right.$ since $\left.\operatorname{var}\left(\mathrm{B}_{\mathrm{i}}\right)=k^{2} \sigma_{b}^{2}+k \sigma_{e}^{2}\right]$

$=2\left(\sigma_{b}^{2}+\sigma_{e}^{2} / k\right)$.

Hence $\operatorname{Var}\left(D_{j^{\prime}-1}\right)=2\left(\sigma_{b}^{2}+\sigma_{e}^{2} / k\right)$ for $j^{\prime}=2,3, \ldots, \mathrm{b}$.

$\operatorname{Cov}\left(D_{1}, D_{2}\right)=\operatorname{Cov}\left(\bar{B}_{1}-\bar{B}_{2}, \bar{B}_{1}-\bar{B}_{3}\right)$

$=\operatorname{Var}\left(\bar{B}_{1}\right)-\operatorname{Cov}\left(\bar{B}_{1}, \bar{B}_{2}\right)-\operatorname{Cov}\left(\bar{B}_{1}, \bar{B}_{3}\right)+\operatorname{Cov}\left(\bar{B}_{2}, \bar{B}_{3}\right)$

$=\sigma_{b}^{2}+\sigma_{e}^{2} / k-0-0+0\left[\right.$ since $\left.\operatorname{var}\left(\mathrm{B}_{\mathrm{i}}\right)=k^{2} \sigma_{b}^{2}+k \sigma_{e}^{2}\right]$

$=\sigma_{b}^{2}+\sigma_{e}^{2} / k$.

Hence

$$
\begin{aligned}
& \operatorname{Disp}(\boldsymbol{D})=\left[\begin{array}{ccc}
2\left(\sigma_{b}^{2}+\sigma_{e}^{2} / k\right) & \cdots & \left(\sigma_{b}^{2}+\frac{\sigma_{e}^{2}}{k}\right) \\
\vdots & \ddots & \vdots \\
\left(\sigma_{b}^{2}+\frac{\sigma_{e}^{2}}{k}\right) & \cdots & 2\left(\sigma_{b}^{2}+\sigma_{e}^{2} / k\right)
\end{array}\right] \text { having order }(\mathrm{b}-1) \times(\mathrm{b}-1) . \\
& =\left(\sigma_{b}^{2}+\frac{\sigma_{e}^{2}}{k}\right)[\boldsymbol{I}+\boldsymbol{J}]=\sigma^{*^{2}} \boldsymbol{W}=\sum(\boldsymbol{D}), \text { say. }
\end{aligned}
$$

Here $\sigma^{*^{2}}=\left(\sigma_{b}^{2}+\frac{\sigma_{e}^{2}}{k}\right), \boldsymbol{I}$ is the identity matrix of order (b-1) and $\boldsymbol{J}$ is the matrix of all elements 1 's; $\boldsymbol{W}=[\boldsymbol{I}+\boldsymbol{J}]$.

Now let us define $\boldsymbol{D}^{*}=\mathrm{k} \boldsymbol{D}$, where $\boldsymbol{D}=\left(D_{1}, D, \ldots, D_{b-1}\right)^{\prime}$ and $\mathrm{k}$ is the constant size of the blocks. Then $\boldsymbol{D}^{*}$ relates to the elementary contrasts among block totals. Our model based on block total contrasts is given by:

$$
\left(\boldsymbol{D}^{*}, \boldsymbol{A} \boldsymbol{\theta}, k^{2} \sigma^{*^{2}} \boldsymbol{W}\right)
$$

where $\boldsymbol{A}$ is the coefficient matrix of $\boldsymbol{\theta}$ of order $(\mathrm{b}-1) \times(\mathrm{v}-1)$ and $\boldsymbol{\theta}=\left(\theta_{1}, \theta_{2}, \ldots, \theta_{v-1}\right)^{\prime}$ with $\theta_{i^{\prime}-1}=\tau_{1}-\tau_{i^{\prime}}, i^{\prime}=2,3, \ldots, \mathrm{v}$; 
$\mathrm{E}\left(\boldsymbol{D}^{*}\right)=\left[\begin{array}{c}\sum_{i=1}^{v}\left(n_{i 1}-n_{i 2}\right) \tau_{i} \\ \sum_{i=1}^{v}\left(n_{i 1}-n_{i 3}\right) \tau_{i} \\ \vdots \\ \sum_{i=1}^{v}\left(n_{i 1}-n_{i b}\right) \tau_{i}\end{array}\right]=\boldsymbol{A} \boldsymbol{\theta}$,

where

$$
\boldsymbol{A}=\left[\begin{array}{ccc}
\mathrm{n}_{22}-\mathrm{n}_{21} & \mathrm{n}_{32}-\mathrm{n}_{31} \ldots & \mathrm{n}_{\mathrm{v} 2}-\mathrm{n}_{\mathrm{v} 1} \\
\mathrm{n}_{23}-\mathrm{n}_{21} & \mathrm{n}_{33}-\mathrm{n}_{31 \ldots} \ldots & \mathrm{n}_{\mathrm{v} 3}-\mathrm{n}_{\mathrm{v} 1} \\
\hdashline \ldots \ldots \ldots \ldots & \ldots \ldots \ldots \ldots \ldots \ldots \ldots \ldots \\
\hdashline \mathrm{n}_{2 \mathrm{~b}}-\mathrm{n}_{21} & \mathrm{n}_{3 \mathrm{~b}}-\mathrm{n}_{31 \ldots} \ldots & \mathrm{n}_{\mathrm{vb}}-\mathrm{n}_{\mathrm{v} 1}
\end{array}\right]
$$

$$
\operatorname{Disp}\left(\boldsymbol{D}^{*}\right)=\operatorname{Disp}(\mathrm{kD})=k^{2} \sum(\boldsymbol{D})=k^{2} \sigma^{*^{2}} \boldsymbol{W}
$$

The Normal Equations for the model (2) are given by

$$
A^{\prime} W^{-1} A \theta=A^{\prime} W^{-1} D^{*}
$$

We may refer to Kshirsagar (1983) for the derivation of (4).

Therefore, BLUE of $\boldsymbol{\theta}$ is

$$
\widehat{\theta}=\left(A^{\prime} W^{-1} A\right)^{-1} A^{\prime} W^{-1} D^{*} \text { where } W^{-1}=(I-J / b)
$$

Further, $\mathrm{E}(\widehat{\boldsymbol{\theta}})=\mathrm{E}\left(\boldsymbol{A}^{\prime} \boldsymbol{W}^{-\mathbf{1}} \boldsymbol{A}\right)^{-\mathbf{1}}\left(\boldsymbol{A}^{\prime} \boldsymbol{W}^{-\mathbf{1}} \boldsymbol{D}^{*}\right)=\boldsymbol{\theta}$

and

$$
\operatorname{Disp}(\hat{\boldsymbol{\theta}})=\mathrm{D}\left\{\left(\boldsymbol{A}^{\prime} \boldsymbol{W}^{-\mathbf{1}} \boldsymbol{A}\right)^{-1} \boldsymbol{A}^{\prime} \boldsymbol{W}^{-\mathbf{1}} \boldsymbol{D}^{*}\right\}=k^{2} \sigma^{*^{2}}\left(\boldsymbol{A}^{\prime} \boldsymbol{W}^{-\mathbf{1}} \boldsymbol{A}\right)^{-1}
$$

The following ANOVA Table is in order.

Table 1: ANOVA table for Inter-block Analysis

\begin{tabular}{ccc}
\hline Source of variation & d.f. & Sums of squares \\
\hline Treatment contrasts & v-1 & $\widehat{\boldsymbol{\theta}}^{\prime}\left(\boldsymbol{A}^{\prime} \boldsymbol{W}^{-\mathbf{1}} \boldsymbol{D}^{*}\right)$ \\
Error & b-v & By subtraction \\
Total & b-1 & $\boldsymbol{D}^{*} \mathbf{W}^{-1} \boldsymbol{D}^{*}$ \\
\hline
\end{tabular}


Remark 1. It must be noted that in the above ANOVA Table, Mean Squares (due to) Error [MSE] provides unbiased estimation of $k^{2} \sigma^{*^{2}}=k^{2}\left(\sigma_{b}^{2}+\frac{\sigma_{e}^{2}}{k}\right)$ with respect to Model (2). This is because the error variance based on block totals/means under MEAM is a linear combination of pure error variance $\left[\sigma_{e}^{2}\right]$ and random block effects variance $\left[\sigma_{b}^{2}\right]$.

Remark 2.When in the model (1) the block effects are treated as fixed effects, the model will change to one involving the fixed block effects and hence no "extra" information will be available for estimation of treatment effects contrasts from the block totals or block total contrasts. This is discussed in the next subsection in the framework of (Fixed effects additive model (FEAM).

\subsection{Fixed Effects Additive Model [FEAM]\& related ANOVA Table}

We now turn back to the same model as in (1) taking $a_{j}$ as the fixed block effect [for each block] so that we are in the framework of FEAM. We first develop a general theory for estimation of treatment effects contrasts based on 'within block contrasts'. For this, we closely follow the use ofC-matrix in the set-up of block designs. We refer to any standard book on ANOVA-based analysis of block designs.

Recall that in its most general set-up of a Balanced Ternary Design, block size ' $\mathrm{k}$ ' has the representation: $\mathrm{k}=\mathrm{t}+\mathrm{qs}$ so that in effect, there are $\mathrm{t}$ distinct treatments each with a single appearance and $\mathrm{s}$ distinct treatments each with replication number $\mathrm{q}$ in each block of the design. Since $q \geq 2$, there are ( $q-1)$ 'pure' errors arising out of these replications for each of the $s$ treatments and hence there are s(q-1) degrees of freedom attributed towards estimation of error variance $\sigma_{e}^{2}$. The computation of corresponding sums squaresis pretty straightforward. Besides, we also have $(\mathrm{t}+\mathrm{s}-1)$ treatment contrasts from within each block. Together, they contribute towards a collection of $b(t+s-1)$ treatment contrasts and only $(v-1)$ treatment contrasts are linearly independent. We are thus led to the identity

$\mathrm{n}-1=\mathrm{b}(\mathrm{t}+\mathrm{sq})-1=\mathrm{bsq}+\mathrm{bt}-1=\mathrm{b}(\mathrm{t}+\mathrm{s}-1)+\mathrm{bs}(\mathrm{q}-1)+(\mathrm{b}-1)$ where

(i) $b(t+s-1)=$ number of within block observational contrasts leading to treatment contrasts and sources of error [for estimation of error variance $\sigma_{e}^{2}$ ];

(ii) $\operatorname{bs}(\mathrm{q}-1)=$ number of within block observational contrasts leading to pure errors [again for estimation of error variance $\left.\sigma_{e}^{2}\right]$;

(iii) $(b-1)=$ number of block total [or block mean] contrasts leading to estimation of treatment effects contrasts [under MEAM] and sources of random block effects error variance together with [observational] error variance [in other words, estimation of $\left.\left(\sigma_{b}^{2}+\frac{\sigma_{e}^{2}}{3}\right)\right]$.

We will now work with the set in (i) to provide intra-block estimates of treatment effects contrasts under FEAM which suggests that only such observational contrasts are relevant. We will end up with ANOVA Table based on Intra-block Analysis.

In standard notations, we refer to the 'Reduced Normal Equations', $\mathbf{C} \boldsymbol{\tau}=\mathbf{Q}$ where

$\mathbf{C}=\mathrm{r} \mathbf{I}-\boldsymbol{N} \boldsymbol{N}^{\prime} / \mathrm{k}$ and $\boldsymbol{N} \boldsymbol{N}^{\prime}$ is as shown in (xvi) above. Further, $\mathbf{Q}=\mathbf{T}-\mathbf{N B} / \mathrm{k}$, $\mathbf{T}$ being the vector of treatment totals and $\mathbf{B}$ being the vector of block totals. The ternary design is balanced and therefore, the $\mathbf{C}$-matrix is completely symmetric [having all diagonal elements equal and also all off-diagonal elements equal]. We have 
$\mathbf{C}=[\mathrm{r}-(\Delta-\lambda) / \mathrm{k}] \mathbf{I}-\lambda \mathbf{J} / \mathrm{k}$ where $\mathrm{r}=$ constant treatment replication number; $\lambda=$ $f_{11}+2 q f_{1 q}+q^{2} f_{q q}$ and $\Delta=\rho_{1}+q^{2} \rho_{q}$. Note that $\boldsymbol{N}^{\prime} \mathbf{1}$ has two representations: $\boldsymbol{N} \boldsymbol{N}^{\prime} \mathbf{1}=\mathrm{k}\left[\rho_{1}+q \rho_{q}\right] \mathbf{1}$ $=\operatorname{rk} \mathbf{1}=[\Delta+(\mathrm{v}-1) \lambda] 1$ and, hence $\mathrm{k} \mathbf{C 1}=[\mathrm{rk}-\Delta-\lambda(\mathrm{v}-1)] \mathbf{1}=\mathbf{0}$. Further, $\boldsymbol{Q}^{\prime} 1=0$.

Therefore, rank $(\mathbf{C})=\mathrm{v}-1$, as expected and the Moore-Penrose g-inverse of $\mathbf{C}$ is easy to work out. Clearly, SS due to Treatment Contrasts $=\boldsymbol{Q}^{\prime} \boldsymbol{C}^{+} \boldsymbol{Q}$ and SS due to pure error $\left[\sigma_{e}^{2}\right]$ derives a contribution from SS within blocks [with $b(k-1) d f$ ] minus SS due to Treatment Contrasts [with $\mathrm{v}-1$ df]. This SS has 2 components: SS due to pure error derived from within treatment $\mathrm{q}$ replications for s treatments in each block [with bs(q-1) df] and SS due to excess of total Within Block SS over SS due to Treatment Contrasts.

Table 2: ANOVA table for Intra-Block Analysis

\begin{tabular}{lcc}
\hline Source of Variation & d.f. & SS \\
\hline Pure Errors & $\mathrm{bs}(\mathrm{q}-1)$ & $\sum_{\mathrm{i}} \sum_{\mathrm{j}} \sum_{\mathrm{u}}\left(\mathrm{y}_{\mathrm{iju}}-\bar{y}_{i j}\right)^{2}$ \\
Treatment Contrasts & $\mathrm{Q}-1$ & $\boldsymbol{Q}$ \\
Errors from within blocks & $\mathrm{b}(\mathrm{k}-1)-\mathrm{bs}(\mathrm{q}-1)$ & $\mathrm{by}$ subtraction \\
Total Within Blocks & $-(\mathrm{v}-1)$ & $\sum \sum_{\mathrm{i}} \sum_{\mathrm{j}}\left(\mathrm{y}_{\mathrm{iju}}-\bar{y}_{i . .}\right)^{2}$ \\
\hline
\end{tabular}

It must be noted that with respect to ANOVA Table 1 for Inter-block Analysis, Total Sum of Squares, shown as $\boldsymbol{D}^{* \prime} \mathbf{W}^{-1} \boldsymbol{D}^{*}$, simplifies to $\sum_{i}\left(y_{i . .}-\bar{y}_{\ldots}\right)^{2}$ which carries (b-1) df. Thus the splitting of Total SS as also of the total degrees of freedom (bk-1) is well understood.

At the last leg, we can work out combined inter- and intra-block estimates of the treatment contrasts. This we do with respect to an illustrative example involving growth of paddy in an agricultural experiment involving 3 treatments.

\section{Combined Intra- and Inter-block estimates}

Here we consider the BTD with parameters: $\mathrm{v}=\mathrm{k}=3, \mathrm{~b}=\mathrm{r}=6, q=2, t=s=1$, $\rho_{1}=2, \rho_{2}=2, f_{11}=0, f_{1 q}=1$ and $f_{q q}=0$. The design has the following block compositions: $\mathrm{B}_{1}=(1,2,2), \mathrm{B}_{2}=(1,3,3), \mathrm{B}_{3}=(2,1,1), \mathrm{B}_{4}=(2,3,3), \mathrm{B}_{5}=(3,1,1), \mathrm{B}_{6}=(3,2,2)$.

We assume the following figures for the yield of paddy [in suitable unit].

Block I: $y_{1}=9.7, y_{2,1}=8.3, y_{2,2}=9.1$

Block III: $y_{2}=9.3, y_{1,1}=9.4, y_{1,2}=10.2$;

Block V: $y_{3}=9.3, y_{1,1}=9.4, y_{1,2}=10.6$
Block II: $y_{1}=9.3, y_{3,1}=8.4, y_{3,2}=9.0$

Block IV: $y_{2}=9.6, y_{3,1}=8.5, y_{3,2}=9.1$,

Block VI: $y_{3}=8.2, y_{2,1}=8.4, y_{2,2}=9.6$

Under the MEAM, we want to explore the contribution of block average contrasts towards treatment effect contrasts. Here we see that there are 5 linearly independent block average contrasts viz., $D_{j-1}=\bar{B}_{1}-\bar{B}_{j} ; \mathrm{j}=2, \ldots, 6$ and there are only 2 treatment contrasts viz., $\boldsymbol{\theta}=\left(\theta_{1}, \theta_{2}\right)^{\prime}$ with $\theta_{1}=\tau_{1}-\tau_{2}, \theta_{2}=\tau_{1}-\tau_{3}$. Further, $\boldsymbol{D}^{*}=3 \boldsymbol{D}$. 
Here $\mathbf{A}=\left(\begin{array}{rr}-2 & 2 \\ -1 & 0 \\ -1 & 2 \\ -2 & 1 \\ 0 & 1\end{array}\right)$ and $\mathbf{W}=\mathbf{I}+\mathbf{J}$ of order 5 . It turns out that

$\widehat{\theta}_{1}=\left[-D_{1}^{*}-D_{2}^{*}+D_{3}^{*}-2 D_{4}^{*}+2 D_{5}^{*}\right] / 6=\mathbf{- 0 . 5 5}$

$\hat{\theta}_{2}=\left[D_{1}^{*}-2 D_{2}^{*}+2 D_{3}^{*}-D_{4}^{*}+D_{5}^{*}\right] / 6=\mathbf{0 . 4 1 6 6}$.

$\operatorname{Disp}(\widehat{\boldsymbol{\theta}})=\frac{3}{2}\left(\begin{array}{ll}2 & 1 \\ 1 & 2\end{array}\right) \sigma^{*^{2}}$ where $\sigma^{*^{2}}=\sigma_{b}^{2}+\frac{\sigma_{e}^{2}}{3}$.

ANOVA Table is displayed below. We find that estimated $\sigma^{*^{2}}$ is given by ERROR MSE/9 = $6.8234 / 27=0.2527$.

Hence, estimated $\operatorname{Var}\left(\hat{\theta}_{1}\right)=3$ times estimated ${\sigma^{*}}^{2}=0.7582=$ estimated $\operatorname{Var}\left(\hat{\theta}_{2}\right)$.

Table 3: ANOVA Table for Inter-block Analysis

\begin{tabular}{ccc}
\hline Source of variation & d.f. & Sums of squares \\
\hline Treatment contrasts & $\mathrm{v}-1=2$ & $\hat{\theta}^{\prime}\left(A^{\prime} W^{-1} \boldsymbol{D}^{*}\right)=1.8099$ \\
Error & $\mathrm{b}-\mathrm{v}=3$ & $6.8234[\mathrm{By}$ subtraction $]$ \\
Total & $\mathrm{b}-1=5$ & $\boldsymbol{D}^{*^{\prime}} \mathrm{W}^{-\mathbf{1}} \boldsymbol{D}^{*}=8.6333$ \\
\hline
\end{tabular}

Turning back to the Intra-block analysis based on FEAM, we have obtained:

$Q_{1}=1.87, Q_{2}=0.07, Q_{3}=-1.94 ; \mathrm{C}=(1 / 3)[12 \mathrm{I}-4 \mathrm{~J}]$;

$\mathrm{C}+=(1 / 12)[3 \mathrm{I}-\mathrm{J}]$;

$\widehat{\boldsymbol{\tau}}=(0.4675,0.0175,-0.4850)^{\prime}$;

(a) $\operatorname{Tr}$. SS $=\hat{\tau}^{\prime} Q=1.8163$ with $2 \mathrm{df}$.

(b) Error SS from within blocks [by subtraction] $=2.8772$ with 4 df.

(c) Further to this, Pure Error SS $=0.32+0.18+0.32+0.18+0.72+0.72=2.34$ with 6 df It follows that (a) - (c) add up to:

(d) Total Within Blocks SS $=\sum_{i}\left[\sum_{j} \sum_{u} y_{i j u}{ }^{2}-k \bar{y}_{i . .}^{2}\right]=4.6935$ with $12 \mathrm{df}$.

Below is the full ANOVA Table.

Table 4: ANOVA Table for Intra-Block Analysis

\begin{tabular}{ccc}
\hline Source of Variation & Df & SS \\
\hline Treatment Contrasts & 2 & 1.8163 \\
Pure Errors & 6 & 2.34 \\
Errors from Within blocks & & 2.8772 \\
Total Within blocks & 12 & 4.6935 \\
\hline
\end{tabular}


From Intra-block Analysis :

$\sigma_{e}^{2}$ estimated $=$ Error SS from the above 2 sources $/(6+4)=[2.8772+2.34] / 10=0.5217$. Finally, based on Intra-Block Analysis, $\hat{\theta}_{1}=\hat{\tau}_{1}-\hat{\tau}_{2}=0.4500 ; \hat{\theta}_{2}=\hat{\tau}_{1}-\hat{\tau}_{3}=0.9525$ with respective estimated variances computed as $\frac{\mathbf{1}}{\mathbf{2}}$ times $\sigma_{e}^{2}$ estimated $=0.2608$. Again, from Inter-block Analysis, $9 \sigma^{*^{2}}=9\left(\sigma_{b}^{2}+\frac{\sigma_{e}^{2}}{3}\right)$ is estimated by the Error MS $=6.8234 / 3=2.2745$. Therefore, $\sigma_{b}^{2}$ estimated $=[2.2745-3 \times 0.5217] / 9=0.0788$.

\section{Combined Intra- and Inter-block Estimates of Treatment Contrasts}

Since the observational contrasts and the block average contrasts are independent, the combined intra-and Inter-block estimation of treatment effects is given by

$$
\hat{\theta}_{c}=\frac{\left(\widehat{\theta}_{1(W B C)} / W 1\right)+\left(\widehat{\theta}_{1(B B C)} / W 2\right)}{(1 / W 1)+(1 / W 2)} .
$$

Hence, $\quad \hat{\theta}_{1, c}=[(-0.55 / 0.7582)+(0.4500 / 0.2608)] /[(1 / 0.7582)+(1 / 0.2608)]=0.1940 ; \hat{\theta}_{2, c}=$ $[(0.4166 / 0.7582)+(0.9525 / 0.2608)] /[(1 / 0.7582)+(1 / 0.2608)]=0.8153$. Further, estimated variance is the same for both and it is given by 0.1940 .

Remark 3: As a final point, it may be noted that it is a trivial exercise to display Combined ANOVA Table for Splitting of Total SS with (bk-1) $=17 \mathrm{df}$. This follows from Tables 3 and 4 .

\section{Acknowledgement}

We express our sincere thanks for administrative co-operation from Dr. K. K. Singh Meitei, Head of the Department of Statistics, Manipur University, Imphal, during exchange visits of the authors. The authors are also grateful to an anonymous referee for his constructive suggestions which helped to improve the presentation of the article.

\section{References}

[1] R.C. Bose, Combined intra-and Inter-block estimation of treatment effects in incomplete block designs. A survey of Statistical designs and linear models (North Holland publishing company, 1975).

[2] E.J.Billington, Balanced n-ary designs: A combinatorial survey and some new results, ARS Combinatoria 17A (1984) 37-72.

[3] E.J. Billington, Designs with repeated elements in blocks:A survey and some recent results, Congressus Numerantium 68(1989) 123-143.

[4] A. M. Kshirsagar, A course in Linear Models. M. Dekker (1983).

[5] M. R. Satam and M. S. Despande, Construction of ternary group divisible designs, J. Ind. Soc. Agril. Statist. 61(3) (2007) 361-363.

[6] C. R. Rao, General methods of analysis in incomplete block designs, Journal of the American Statistical Association 42 (1947) 541-561.

[7] H.L. Sharma, R.N. Singh and R. Tiwari, Balanced ternary, ternary group divisible and nested ternary group divisible designs, Ind. Soc. Agril.Statist. 67(3) (2013) 339-344. 
[8] S. R. Searle, Inter- and intra- block estimation of treatment effects in randomized blocks. Technical report BU-897M (1986) in the Biometrics Unit.

[9] K.D. Tocher, The design and analysis of block experiments (with discussion), J. Roy. Statist. Soc. Ser. B 14 (1952) 45-100.

[10] F. Yates, The recovery of inter block information in varietal trials arranged in three dimensional lattices, Ann. Eugenics 9 (1939) 136-156.

[11] F. Yates, The recovery of inter block information in balanced incomplete block designs, Annals of Eugenics 10 (1940) 317-325. 